\title{
Long and narrow Josephson tunnel junctions of mixed overlap and inline character
}

\author{
Olsen, O.H.; Samuelsen, Mogens Rugholm
}

Published in:

Journal of Applied Physics

Link to article, DOI:

$10.1063 / 1.331883$

Publication date:

1983

Document Version

Publisher's PDF, also known as Version of record

Link back to DTU Orbit

Citation $(A P A)$ :

Olsen, O. H., \& Samuelsen, M. R. (1983). Long and narrow Josephson tunnel junctions of mixed overlap and inline character. Journal of Applied Physics, 54(11), 6522-6525. https://doi.org/10.1063/1.331883

\section{General rights}

Copyright and moral rights for the publications made accessible in the public portal are retained by the authors and/or other copyright owners and it is a condition of accessing publications that users recognise and abide by the legal requirements associated with these rights.

- Users may download and print one copy of any publication from the public portal for the purpose of private study or research.

- You may not further distribute the material or use it for any profit-making activity or commercial gain

- You may freely distribute the URL identifying the publication in the public portal

If you believe that this document breaches copyright please contact us providing details, and we will remove access to the work immediately and investigate your claim. 


\title{
Long and narrow Josephson tunnel junctions of mixed overlap and inline character
}

\author{
O. H. Olsen \\ NIRO Atomizer, Research and Development System and Design Division, Gladsaxevej, DK-2860 Soeborg, \\ Denmark
}

M. R. Samuelsen

Physics Laboratory I, The Technical University, of Denmark, DK-2800 Lyngby, Denmark

(Received 11 March 1983; accepted for publication 19 April 1983)

\begin{abstract}
A model describing long Josephson junctions of mixed overlap and inline geometry is presented. The shape of the first zero field step is calculated for this model using a perturbation approach. The question of influence of external magnetic field on the maximum supercurrent is investigated for overlap, inline, and mixed overlap-inline geometries. A linear dependence is found for the inline model and for mixed overlap-inline junctions in agreement with experiments.
\end{abstract}

PACS numbers: 74.50. + r, 84.40.Mk, 85.25. + k

\section{INTRODUCTION}

Josephson junctions have recently attracted much theoretical and experimental interest ${ }^{1,2}$ because of their various potential applications as, e.g., microwave generators in high frequency integrated circuits, ${ }^{3,4}$ and data processing systems. ${ }^{5}$ Most of the experimental findings concerning Josephson junctions such as the appearance of dc singularities in the IV characteristics and the accompanied emitted radiation have been explained in the framework of fluxon dynamics. ${ }^{6}$ The fluxons are quantized magnetic vortices present in Josephson junctions whose motion is assumed to be governed by the perturbed sine-Gordon equation. The singularities in the IV characteristics are due to resonant motion of fluxons along a Josephson junction with reflections in both ends.

Long Josephson junctions are often assumed to be of either overlap or inline geometry, which however, both represent idealized situations. A real Josephson junction will typically-owing to geometrical irregularities-be a mixture of these two. The aim of the present work is to present a simple model which makes it possible to examine junctions of mixed type. For this model we investigate the influence of an external magnetic field on the supercurrent. Experiments show ${ }^{7.8}$ that the supercurrent for long junctions decreases linearly for increasing external magnetic field. The theory for the pure inline geometry predicts this result while the theory for the overlap geometry junction predicts a nonlinear dependence. However, the mixed overlap-inline model yields the correct result in almost all cases. Further, we calculate the form of the first zero field step obtained for this model.

This paper is structured as follows: Sec. II describes the models for overlap, inline, and mixed overlap-inline geometry junctions, and presents the shape of the first zero field step. Section III contains expressions relating the supercurrent to the external magnetic field.

\section{MODELS}

It is a well known assumption that the equation describing fluxon motion is the perturbed sine-Gordon equation":

$$
\phi_{x x}-\phi_{t t}=\sin \phi+\alpha \phi_{\mathrm{e}}+\eta,
$$

with appropriate boundary conditions. Here, $\phi(x, t)$ is the space and time dependent phase difference between the two superconduction films. The spatial variable $x$ is measured in units of the Josephson penetration depth $\lambda_{J}=\left(\hbar / 2 \mu_{0} e d J\right)^{1 / 2}$ and the time $t$ in units of the reciprocal plasma frequency $\omega_{0}^{-1}$, where $\omega_{0}=(2 e J / \hbar C)^{1 / 2}$. Here, $J$ is the maximum pair current density, $d$ the magnetic thickness of the barrier $\left(d=2 \lambda_{L}+t_{0}\right)$, and $C$ the capitance per unit area. The parameter $\alpha$ describes dissipative effects, $\alpha=G(\hbar / 2 e J C)^{1 / 2}$, where $G$ is the shunt conductance per unit area. The damping parameter is related to the McCumber parameter $B_{C}$ by $\alpha=1 / \sqrt{\beta_{C}} \cdot \eta$ represents a uniformly distributed bias current and depends on $I_{\mathrm{dc}}$ and the geometry.

In the following we descirbe the models ${ }^{10}$ for overlap, inline, and mixed overlap-inline geometry.

\section{A. Overlap geometry}

In the overlap geometry the bias current distribution is uniform along the junction; thus, $\eta=\eta_{0}=I_{\mathrm{dc}} / J w L$, where $L$ and $w$ are the length and width of the junction. One-dimensional motion of the fluxons requires the assumptions $l=L / \lambda_{J}>1$ and $w / \lambda_{J}<1$. No self-fields are present at the junction ends; therefore, the boundary conditions are

$$
\phi_{x}(0, t)=\phi_{x}(l, t)=0,
$$

and consequently, no energy input occurs at the ends. The fluxon velocity is determined as a balance between the energy dissipation and the energy input through the bias current. At the boundaries the fluxon is converted into an antifluxon by an almost elastic reflection and a steady-state motion is obtained at the average normalized voltage $V_{1}$, of the first zero field step:

$$
V_{1}=\frac{2 \pi u_{\infty}}{l}=\frac{2 \pi}{l}\left[1+\left(\frac{4 \alpha}{\pi \eta}\right)\right]^{-1 / 2},
$$

where $u_{\infty}$ is the power balance velocity.

\section{B. Inline geometry}

The dynamic picture in the inline case is quite different. The bias current is distributed antisymmetrically and thus $\eta=0$. Instead self-fields occur in both ends giving rise to the boundary conditions 


$$
\phi_{x}(0, t)=-\phi_{x}(l, t)=\kappa
$$

where $\kappa=\kappa_{0}=\left(I_{\mathrm{dc}}\right) /\left(2 w J \lambda_{J}\right)$. In this case the self-field provides an energy input of

$$
\Delta E=\frac{\pi \kappa}{2}
$$

as a result of a reflection of a fluxon into an antifluxon. This is because the reflection corresponds to a phaseshift of $4 \pi$. The energy is measured in units of the fluxon rest energy $E_{0}=8 \hbar / w \lambda_{J} / 2 e$.

Thus, for the inline case the physical mechanism is that the fluxon gets a kick at one junction end, and its velocity decays towards zero as it propagates towards the other end where it gets another kick to maintain a steady-state motion. Integrating the fluxon trajectory the result for the average velocity $u_{a v}$ and the normalized voltage of the zero field step is given by ${ }^{11}$

$$
\frac{\pi \kappa_{0}}{2 \alpha l}=\frac{\frac{\sinh (\alpha l / 2)}{(\alpha l / 2)}}{\left[\left(\frac{\tanh \left(\alpha l / 2 u_{a v}\right)}{\tanh (\alpha l / 2)}\right)^{2}-1\right]^{1 / 2}} .
$$

Again, $V_{1}=(2 \pi / l) u_{a v}$ and $\kappa_{0}=I_{\mathrm{dc}} / 2 \lambda_{J} w J$. For $\alpha l \rightarrow 0 \mathrm{Eq}$. (6) becomes identical to Eq. (3).

\section{Mixed overlap-inline geometry}

In the mixed overlap-inline geometry model we assume the junction to be a mixture of the two former geometries. Let a fraction $y$ of the total bias current $I_{\mathrm{dc}}$ be of overlap type and thus the fraction $(1-y)$ of inline type. Then $\eta$ in Eq. (1) becomes

$$
\eta=y \cdot \eta_{0}
$$

and $\kappa$ in Eqs. (4) and (5)

$$
\kappa=(1-y) \kappa_{0}=(1-y) \frac{\eta_{0} l}{2} .
$$

In order to determine the shape of the zero field step in the mixed overlap-inline geometry model we follow the procedure in Ref. 11 where the result in Eq. (6) was obtained by application of a perturbation approach. The dynamic picture in the mixed case is different from the former examples. As a result of the reflection the fluxon gets an energy increase due to the "inline function" of the bias current given by Eqs. (5) and (7b). While the fluxon travels in junction the velocity decreases towards the power balance velocity given by Eqs. (3) and (7a). The fiuxon trajectories are symmetric with respect to $l / 2$. In order to calculate the trajectories we introduce the notation ${ }^{11}$

$$
\begin{aligned}
& u=\tanh a, \\
& z=u \gamma(u)=P_{f},
\end{aligned}
$$

where $u=u(x, t)$ is the velocity of the fluxon, $\gamma(u)$ the Lorentz factor $\left(1-u^{2}\right)^{-1 / 2}$, and $P_{f}$ the normalized momentum of the fluxon. From Eq. (8) it follows that

$$
\begin{aligned}
& \gamma(u)=\cosh a, \\
& z=\sinh a, \\
& z_{\infty}=\pi \eta / 4 \alpha, \\
& u_{\infty}=\tanh a_{\infty} .
\end{aligned}
$$

The equation of motion for a single fluxon in the perturbed sine-Gordon model is ${ }^{9}$

$$
\frac{d P_{f}}{d t}=-\alpha P_{f}+\frac{\pi \eta}{4} .
$$

Now, the energy resulting from a reflection can be written

$$
\gamma\left(u_{2}\right)-\gamma\left(u_{1}\right)=\Delta E,
$$

or

$$
\cosh a_{2}-\cosh a_{1}=\Delta E,
$$

where $\Delta E$ is given by Eqs. (5) and (7b); $u_{1}$ is the velocity of the incoming fluxon and $u_{2}$ the velocity of the reflected antifluxon. Integration of Eq. (10) yields the momentum of the fluxon as a function of the time which can be solved to give the time of flight

$$
t_{12}=\ln \frac{\sinh a_{1}-\sinh a_{\infty}}{\sinh a_{2}-\sinh a_{\infty}},
$$

for the fluxon.

One more integration of Eq. (10) yields the trajectory for the fluxon which introduces the length of the junction

$$
\frac{\alpha l}{u_{\infty}}=\alpha t_{12}-\frac{a_{1}-a_{2}}{a_{\infty}}+2 \ln \frac{\cosh \frac{a_{2}+a_{\infty}}{2}}{\cosh \frac{a_{1}+a_{\infty}}{2}} .
$$

Here $u_{\infty}$ is given by Eqs. (3) and (7a).

The two unknowns $a_{1}$ and $a_{2}$ can now be found by solution of the two Eqs. (11) and (13). The voltage of the zero field step is given by the average velocity $u_{a v}$ defined by $u_{a v}=l / t_{12}$. Because of the complexity of the equations we have not been able to find a closed expression for this quantity. However, a numerical solution has been performed. This is shown in Fig. 1(a) for $\alpha l=1$ and Fig. 1(b) for $\alpha l=2$. The lower curves are obtained from Eq. (3) corresponding to $y=1$ (pure overlap case) while the upper curves are obtained from Eq. (6) corresponding to $y=0$ (pure inline case). It is seen that as long as $y$ is larger than 0.5 the first zero field step is very close to the pure overlap case. Finally, we remark that we have not been able to follow the zero field step to $u_{a v}=0$ in the numerical solution (where the zero field step must start for $y \neq 0$ ).

\section{INFLUENCE OF EXTERNAL MAGNETIC FIELD ON THE SUPERCURRENT}

In this section we calculate expressions which describe the influence of an external magnetic field on the maximum supercurrent. The supercurrent is the zero-voltage current, i.e., the solution to Eq. (1) with the condition $\phi_{t}(x, t)=0$. Applying an external magnetic field to a long Josephson 

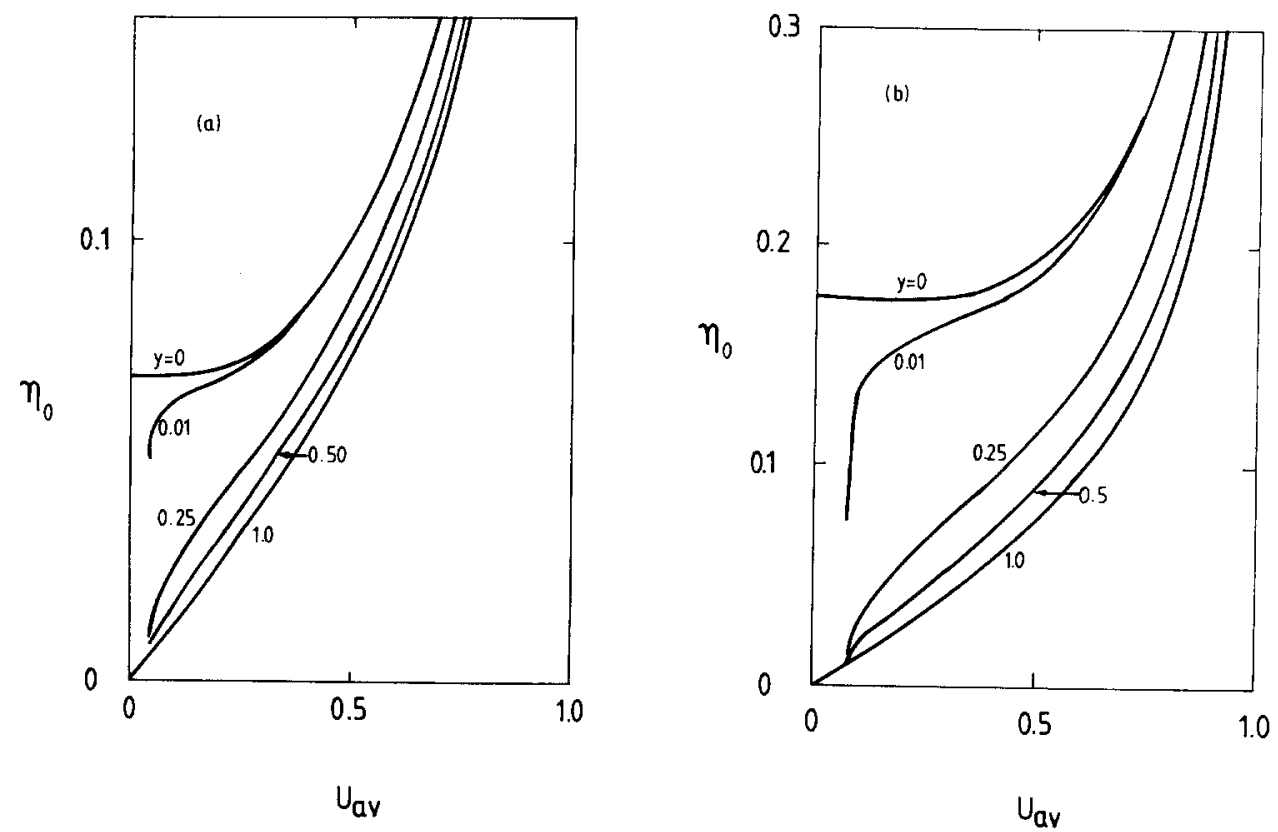

FIG. 1. First zero field step for mixed overlap-inline geometry. Normalized current $\eta_{0}$ vs average velocity $u_{a v}$ (voltage). Parameters: $\alpha l=1$ (a) and $\alpha l=2$ (b). $y=0$ corresponds to pure inline geometry and $y=1$ corresponds to pure overlap geometry.

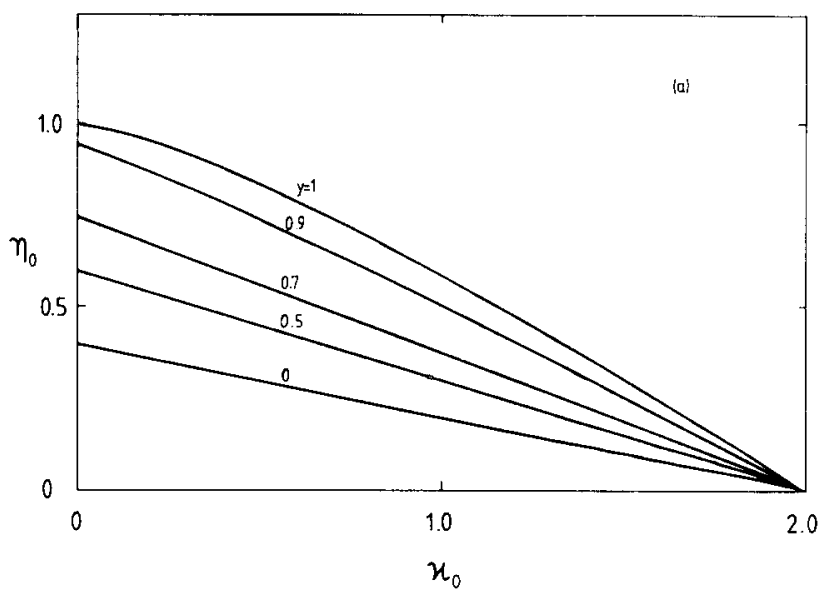

junction will reduce the magnitude of the supercurrent which can be forced through the junction. ${ }^{7,8}$ The expressions are given for the three models in the former section.

For the inline junction an external magnetic field will change the boundary conditions in Eq. (4) to

$$
\phi_{x}(0, t)=-\phi_{x}(l, t)+2 \kappa_{e x t}=\kappa+\kappa_{e x t},
$$

where $\kappa_{\text {ext }}$ is the applied magnetic field normalized to $\lambda_{J} J$. The magnetic field can be regarded as a part of a stationary fluxon from the end into the junction. Since the maximum value of $\phi_{x}$ for a stationary fluxon is two, the relation for a long inline junction between the maximum supercurrent and the applied magnetic field then is

$$
\kappa_{\max }=2-\kappa_{e x t},
$$

or

$$
\eta_{\max }=\frac{I_{\max }}{w L J}=\frac{4}{l}-\frac{2 \kappa_{\text {ext }}}{l},
$$

which is a straight line in agreement with experiments. ${ }^{\text {? }}$

For a long overlap junction the maximum supercurrent can be found by solving Eq. (1) with $\phi_{x}(0, t)=\kappa_{e x t}$ and $\phi_{t}(x, t)=0$. The trivial solution is

$$
\phi_{0}(x, t)=-\sin ^{-1} \eta(\bmod 2 \pi) .
$$

Integration of Eq. (1) using $\phi_{t}(x, t)=0$ yields

$$
\frac{1}{2} \phi_{x}^{2}=n \phi-\cos \phi-\eta \phi_{0}+\cos \phi_{0}=F(\phi) .
$$

Here, $F(\phi)$ can be considered as a potential having stable points in $\phi_{1}=\phi_{0}+2 n \pi$ and unstable points in $\phi_{2}=\pi-\phi_{0}+2 n \pi$. Now, if the left side of Eq. (17) exceeds the energy gab $F\left(\phi_{2}\right)-F\left(\phi_{1}\right)$ the phase will start to increase. Thus, the expression for the maximum supercurrent is ${ }^{12}$

$$
\left(\frac{\kappa_{e x t}}{2}\right)^{2}=\sqrt{1-\eta_{\max }^{2}}-\eta_{\max } \sin ^{-1} \sqrt{1-\eta_{\max }^{2}} .
$$

FIG. 2. Supercurrent $\eta_{\max }$ vs the applied magnetic field $\kappa_{\mathrm{exx}}$. Parameters: $l=10(\mathrm{a}), l=20$ (b). $y=0$ corresponds to pure inline geometry and $y=1$ corresponds to pure overlap geometry.

Here, we have assumed that the junction is half infinite.

The relation Eq. (18) between $\kappa_{e x t}$ and $\eta_{\max }$ is certainly 
not linear in contrary to experiments. [The upper curves in Figs. 2(a) and 2(b)].

The mixed overlap-inline case is obtained by replacing $\kappa_{\text {ext }}$ with $\kappa_{\text {ext }}+\kappa_{0}(1-y)$ and $\eta_{\max }$ with $\eta_{0, \max } y$ in Eq. (18), [see Eq. (7)]. In Figs. 2(a) and 2(b) we show the supercurrent versus external magnetic field given by the mixed overlapinline model. The parameters are $l=10$ [Fig. 2(a)] and $l=20$ [Fig. 2(b)]. It is seen that for $y \leq 0.9$ the curves are almost straight lines in agreement with experiments. The lower curves in Figs. 2(a) and 2(b) show Eq. (15b).

\section{CONCLUSION}

In the present paper we have presented a model for long Josephson junctions of mixed overlap-inline geometry based on a mixture of the well-known models for pure overlap and inline geometry junctions.

Firstly, we have examined the shape of the first zero of field step for this model using a perturbation method. The shape only differs slightly from the result obtained from the pure overlap geometry model if the mixing parameter is larger than 0.5 .

Secondly, relations describing the maximum supercurrent in the presence of an external magnetic field have been presented for pure overlap, pure inline, and mixed overlapinline geometry models. The mixed overlap-inline geometry model shows a linear relation if the mixing parameter is less than 0.9 .

'P. S. Lomdahl, "Soliton dynamics in nonintegrable sine-Gordon systems," DCAMM Rep. No. S20 (1982), The Technical University of Denmark.

${ }^{2}$ N. F. Pedersen, "Solitons in long Josephson junctions" in Nato advanced study institutes series, Proceedings of the 1982 Summerschool, Erice, Italy (Plenum, New York, to be published).

${ }^{3}$ S. N. Erné and R. D. Parmentier, J. Appl. Phys. 51, 5025 (1980).

${ }^{4}$ S. N. Ernê and R. D. Parmentier, J. Appl. Phys. 52, 1608 (1981).

${ }^{5}$ T. V. Rajeerakumar, IEEE Trans. Magn. MAG-17, 591 (1981).

${ }^{6}$ E. Joergensen, V. P. Koshelets, R. Monaco, J. Mygind, M. R. Samuelsen, and M. Salerno, Phys. Rev. Lett. 49, 1093 (1982).

${ }^{7}$ Klaus Schwidtal, Phys. Rev. B 2, 2526 (1970).

${ }^{8}$ G. Costabile, A. M. Cucolo, S. Pace, R. D. Parmentier, B. Savo, and R. Vaglio, in SQUIDS and Their Applications, edited by H. D. Hahlbohm and H. Lubbig (de Gruyter, Berlin, 1980), p. 147.

${ }^{9}$ D. W. McLaughlin and A. C. Scott, Phys. Rev. A 18, 1652 (1978).

${ }^{10}$ B. Dueholm, E. Joergensen, O. A. Levring, R. Monaco, J. Mygind, N. F. Pedersen, and M. R. Samuelsen, IEEE Trans. Magn., Proc. ASC82 (to be published).

${ }^{11}$ O. A. Levring, N. F. Pedersen, and M. R. Samuelsen, J. Appl. Phys. 54, 987 (1983).

${ }^{12}$ A. F. Volkov (private communication). 